\title{
Construction of Multi-point Quality Analysis Model of Tobacco Industry Co-production Based on Integrated "Platform + Module"
}

\author{
Huali Zheng*1 \\ ${ }^{1}$ Production Management Department China tobacco Zhejiang Industrial Co., Ltd, Hangzhou, China
}

\begin{abstract}
Under the multi-point production management mode, Researchers establish a unified cooperative production multi-point product quality analysis standard, set benchmarks, compare performance, create a process quality management atmosphere of ratio, learning, catching up and exceeding, so that multi-point quality management ability can be obtained together to improve, product quality has been continuously improved. At present, the quality analysis of multi-point processed products in cooperative production is only a simple average and standard deviation detection and comparison of key processes and individual indicators, or a single analysis of the appearance quality of cigarettes. The analysis is relatively isolated and no in-depth research is conducted. It is impossible to comprehensively and comprehensively compare the processing quality levels of various processing enterprises. In response to these problems, this paper compares and analyzes several quality controlling schemes, selects the "platform + module" scheme and designs and constructs it. The article discusses the specific implementation measures of the design plan. From the perspective of the implementation effect of the plan, it can provide guarantee for improving the production quality of cigarettes.
\end{abstract}

\section{Introduction}

With the development of inter-provincial cooperative production of Zhejiang China Tobacco, multi-point processing of the same brand has become a common phenomenon. Homogenization is a requirement to ensure the consistency and quality stability of multi-point processing of cigarette brands. It is also the industry's organizational structure adjustment and adaptation. As the cooperative production sites are scattered, the production capacity gap is large, and the quality control level is uneven. The analysis method of each processing point in the previous period did not consider the importance of each indicator. The analysis of each indicator is not comprehensive enough, and the consideration is not perfect and sufficient. To this end, the joint production department focuses on lean improvement, through the application of lean concepts, ideas, methods and tools, scientific analysis of production processes, process indicators and quality control structure and other factors to help standardize the management of the overall production process and further identify and eliminate process processes The current situation of data is complex and redundant, and a quality analysis model of multi-point cigarette processing products is established. This article briefly analyzes the connotation of cooperative production quality analysis, and discusses the comparative analysis of the quality analysis management operation process, aiming at the actual quality data management system operation process. Based on production lines at various points, this paper establishes analysis models for package, wire, and material to comprehensively evaluate the physical measurement, appearance, internal quality, and overall quality level of the same product processed in multiple production enterprises, providing for the improvement of homogenized production level. Support to provide a strong guarantee for the comprehensive improvement of product quality and process level [1].

\section{Research the basic route}

The content of the current cooperative production includes the inspection report and data collation of the raw and auxiliary materials of the partner, the key indicators of each batch of the silk produced by the processor, and the sequenced tobacco data (moisture content, filling value, finishing rate, shredded rate of cut tobacco), a collection of online inspection data and appearance inspection data for packaging and rolling, as well as inspection data from the processing party, monthly inspection data from quality supervision inspection stations and market complaint data. Cooperative production does not establish a set of analysis standards for the correlation between a series of indicators such as upper and lower processes [2].

The basic idea of the project includes combing the current quality analysis methods of cooperative production in a process method, and trying to design an integrated management system.

The main problems to be solved include taking the cooperative production unit as the main unit and the responsible unit, distinguishing the weight according to the responsibility of the controllable index, and establishing the index analysis system [3]. 
The evaluation indicators include the monthly monitoring and inspection summary of the testing station, the quality stability of the wire making process, the process execution stability summary, the package online supervision summary, the processing party's warehouse and reordering inspection summary, the market information feedback summary, and the processing points. The score is accumulated for comprehensive evaluation. The evaluation indicators are individual, sub-process, submodule weight, setting and calculation formulas. The overall data process design chart analysis is used to identify opportunities for improvement. A comprehensive analysis of the efficiency waste in the process is carried out, and lean tools and methods are used to improve the direction, rearrange the process [4].

\section{Choice of research plan}

- $\quad$ Scheme one, add process monitoring personnel and feedback product quality information. Easy to implement; large wage expenses; It can increase the sense of the scene, but the personnel must be on tour at all times; there are many process points, and the range of process monitoring personnel is large; It requires the coordination of relevant departments to arrange process monitoring personnel [5].

- Scheme two, obtain product quality information with the help of real-time monitoring system data acquisition curve. The scheme is easy to implement and the curve has good real-time performance, but the curve cannot be converted to a data format, and analysis is relatively difficult; It is necessary to arrange high-quality data analysis related personnel; its data record availability is poor.

- Scheme three, the construction and application of an integrated "platform + module" cooperative production multi-point product quality analysis model. It needs to be developed independently, but it needs personnel familiar with on-site data collection and analysis and software design at the cooperative production site; It does not need to add any personnel, the system can automatically complete data analysis and storage; In the system design process, we can design real-time and effective statistical methods according to actual needs; The system development is relatively independent and does not affect daily production tasks.

The quality analysis system based on the cooperative production process needs to be able to realize real-time data analysis and processing; The relevant personnel of cooperative production need to obtain the current production process and historical quality information conveniently, quickly and accurately through the system. Therefore, we choose option three [6].

\section{Program development}

The design of the statistical system is based on the realtime monitoring system, which will process the data acquisition data and write it into the data acquisition database. The VB function reads the system data from the real-time monitoring, the front-end application processes the statistical data and stores it in the database, and the VB function stores the statistical results to the database [7].

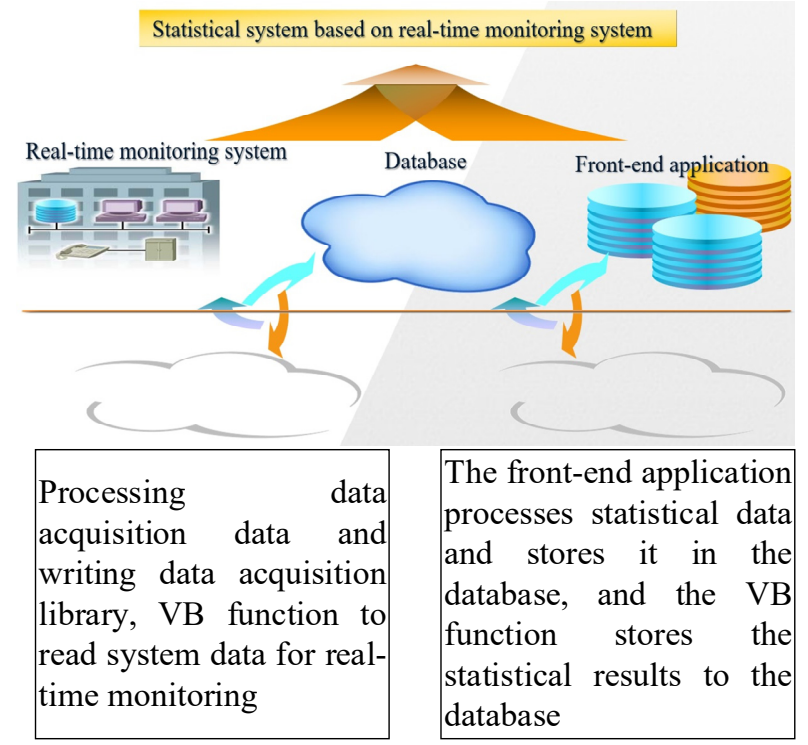

Fig1. Integrated "platform + module" design

The system is mainly divided into four parts.

\subsection{Build database.}

It is used to store data and statistical results. Install database development software, establish data acquisition database and statistical result database, establish user data table (data acquisition table and general data table), define user stored procedures and triggers, and base statistical result database on workshop data management system database based on data acquisition database in.

\subsection{Configure SQL.}

Relevant information to establish a connection between the on-site process point and the database. Install SQL, collect operation points, connect to the database, establish associations, collect all operation point information, configure the operation points to be collected in SQL, and configure the connection between SQL and the database.

\subsection{Design front-end applications.}

Make the system have statistical functions, and visual output. Design front-end tools, develop statistical functions, develop database interfaces, program debugging, allocate and implement the main framework of the application and each module, after the development of each module, integrate the system and debug. 


\subsection{System testing and improvement.}

Check the overall function and performance of the system, and improve the deficiencies, check the reliability of the connection between the front-end application and the database, and test the system performance.
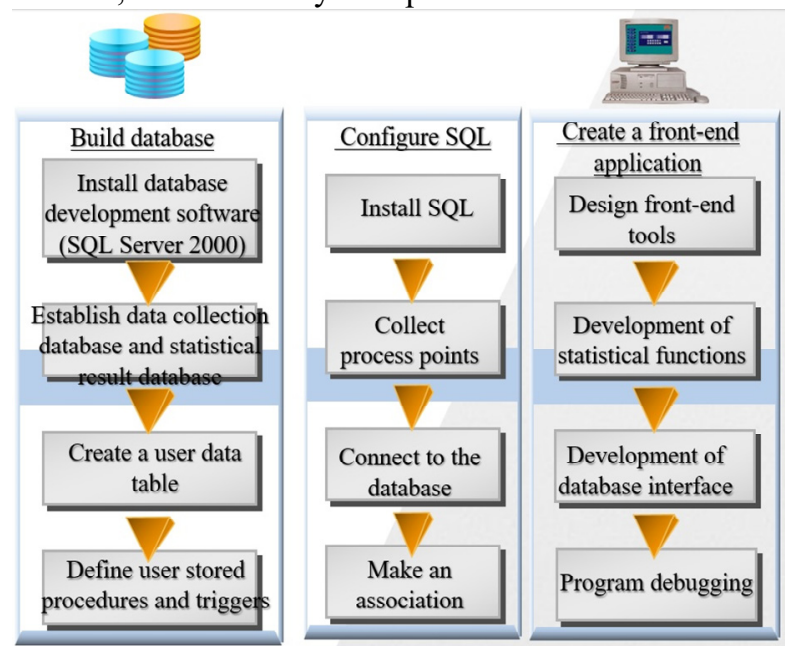

Fig2. Development strategy of integrated "platform + module"

\section{Implementation of the scheme}

Set up four modules of digital mining view, silk production process monitoring, wrapping process monitoring, finished product moisture monitoring, cigarette appearance defects. It is divided into six steps [8]

- $\quad$ Step 1, Create a new configuration. Specify a path for storing configuration data files, and select the control connection service for data transmission.

- $\quad$ Step 2, Define connectors. Verify the computers and users, used to run each connection service. There must be a connection configuration for each connection service in step one.

- $\quad$ Step 3, Define data points. Refer to the control connector defined in step 2, and determine the data points that need to be collected from the control system, including data reading and dumping, application function modules (database call function statistical functions, etc.), Including the call of basic data and the storage of statistical results.

- $\quad$ Step 4, define the data object. Reference the data control connector defined in step two, and determine that the column information or stored procedure of the data table needs to be referenced during data transmission.

- Step 5, Configure the transmission. Define the binding of the control system acquisition point in step three to the data table or stored procedure in step four in each transmission, and define the conditions for transmitting trigger events and storing data.

- $\quad$ Step 6, Verify the selected transmission on the database configuration, software functions, and system operating speed.

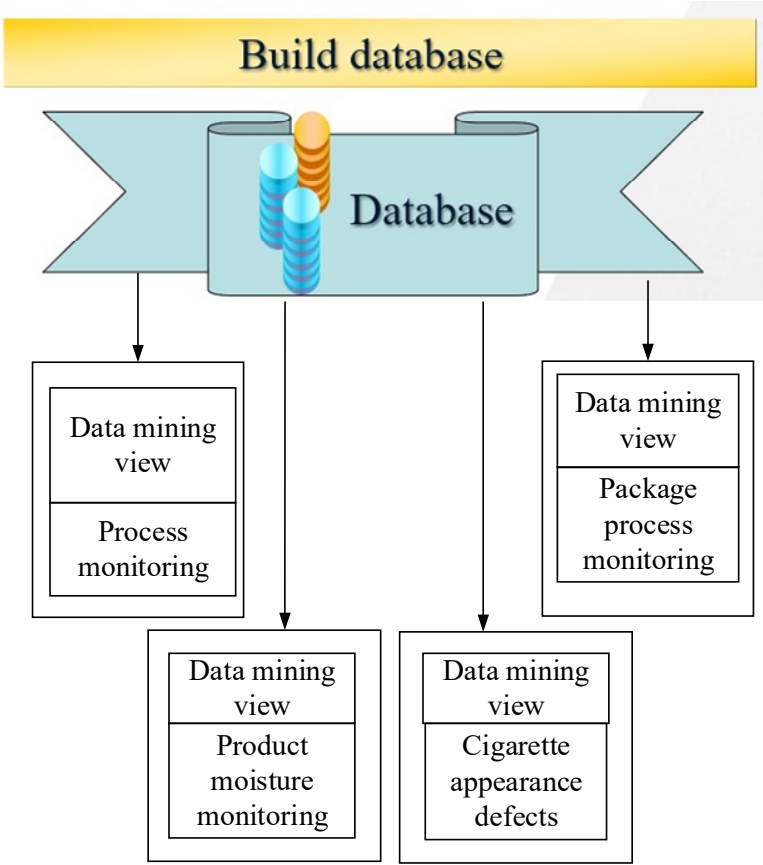

Fig3. Building a database based on cigarette products

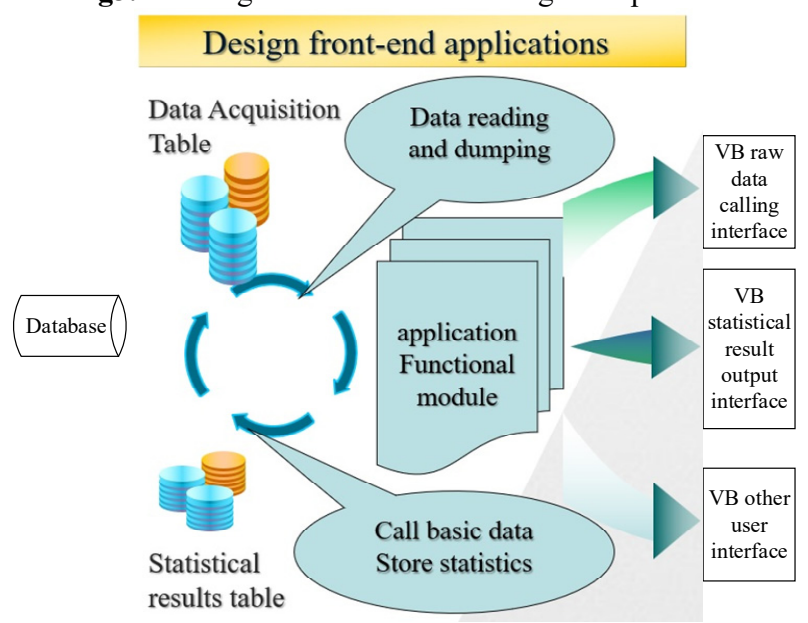

Fig4. Front-end application design

\section{Effectiveness of using the plan}

From the perspective of the operation of the system, in the cooperative production process, the system can realize real-time data collection and processing of package and silk-making processes. Production quality related personnel can easily, quickly, and accurately obtain the process and historical quality of the work-in-progress through the system information. The application effect is reflected in the following aspects.

\subsection{Operability}

After the platform system is established, the process quality analysis can be quickly and intuitively fed back to the operator. While understanding the process quality, it can make analysis and diagnosis for the single process of silk production or the single sample of the package, and through continuous accumulation, the purpose of standardizing the production operation of the process is achieved. 


\subsection{Real-time}

For the production of each batch of each group of samples, more analysis of the collected data can be obtained. For a single batch of single samples, during the production process, data statistics can also be made for the batch or sample.

\subsection{Practicality}

The quality information of the work-in-process process acquired by the statistical system during the production process of the single batch of silk or the sample of the wrapped package has a good guiding significance for the operator. The operator can adjust the process control parameters or physical measurement parameters according to the process statistical results.

\subsection{Availability}

The operation process of the system is a process of data accumulation. The accumulation of quality data of each brand for a long period of time can be used to explore the control parameter setting method of different brands at a certain process point, as well as the specific situation of the physical index of the package of different brands.

\subsection{Promotional.}

Due to the high practicality of the system, this system has a high value of promotion under the situation of implementing statistical process control management of quality process control in the cooperative production team.

\section{Conclusion}

The project is based on an integrated "platform + module" to build a multi-point product quality analysis model for cooperative production with applications. The most distinctive feature is the real-time and practicality, which usually guarantees the analysis of batch and sample data. The project introduces advanced computer technology and network communication technology in the field of automatic control, and builds a quality analysis system suitable for the cooperative production process. Among them, its technology includes database development and management technology, database configuration and configuration technology, etc.

\section{References}

1. F. Zou, Research on high-quality development of tobacco import and export enterprises. Modern Trade Industry, 2020, vol.41, no.17, pp.21-22.

2. X.L. Wang, H. Yang, Y Ruan, Analysis of Intellectual Property Management of Tobacco Commercial Enterprises under Reform and Innovation. China Collective Economy, 2020, no.15, pp.122-123.

3. Y.H. Lu, G.X. Zhang, J. Meng, et al., Research on the raw material processing characteristics of Shandong flue-cured tobacco in air drying process. Anhui Agricultural Science Bulletin, 2016, vol.22, no.15, pp.124-128.

4. N. Li, Research on the quality diagnosis technology of tobacco silk-making process based on neural network. Zhejiang University of Technology, 2016.

5. F.Q. Du, C.Z. Qian, D.F. Tang, et al., Calculation and application of sampling characteristic values of tobacco materials for inspection. Tobacco Science and Technology, 2013, no.4, pp.9-11.

6. W.T. Gou, Strengthen brand cooperative production to ensure the healthy development of the tobacco industry brand. Modern Business, 2013, no.10, pp.89.

7. X.D. Lu, Research on the application of data warehouse technology in tobacco production management. Tongji University, 2006.

8. J. Tang, L.D. Cheng, L. Zhang, Statistical technology analysis of cigarette single weight. Tobacco Science and Technology, 2000, no.3, pp.9-11. 